ARTICLE

Received 29 Jun 2014 | Accepted 12 Nov 2014 | Published 19 Dec 2014

DOI: $10.1038 /$ ncomms6841

\title{
Microwave gain medium with negative refractive index
}

Dexin $\mathrm{e}^{1,2}$, Kihun Chang ${ }^{2}$, Lixin $\operatorname{Ran}^{1} \&$ Hao Xin ${ }^{2,3}$

Artificial effective media are attractive because of the fantastic applications they may enable, such as super lensing and electromagnetic invisibility. However, the inevitable loss due to their strongly dispersive nature is one of the fundamental challenges preventing such applications from becoming a reality. In this study, we demonstrate an effective gain medium based on negative resistance, to overcompensate the loss of a conventional passive metamaterial, meanwhile keeping its original negative-index property. Energy conservation-based theory, full-wave simulation and experimental measurement show that a fabricated sample consisting of conventional sub-wavelength building blocks with embedded microwave tunnel diodes exhibits a band-limited Lorentzian dispersion simultaneously with a negative refractive index and a net gain. Our work provides experimental evidence to the assertion that a stable net gain in negative-index gain medium is achievable, proposing a potential solution for the critical challenge current metamateiral technology faces in practical applications.

\footnotetext{
${ }^{1}$ Laboratory of Applied Research on Electromagnetics (ARE), Zhejiang University, Hangzhou 310027, China. ${ }^{2}$ Department of Electrical and Computer Engineering, University of Arizona, Tucson, Arizona 85721, USA. ${ }^{3}$ Department of Physics, University of Arizona, Tucson, Arizona 85721, USA. Correspondence and requests for materials should be addressed to H.X. (email: hxin@ece.arizona.edu) or to L.R. (email: ranlx@zju.edu.cn.).
} 
A rtificial effective media are attractive because of the fantastic applications such as super lens and electromagnetic invisibility ${ }^{1,2}$. However, the inevitable loss due to their strongly dispersive nature is one of the fundamental challenges preventing such applications from becoming a reality. Recently, the discussions of incorporating active elements into artificial media to compensate loss and potential bandwidth have attracted more and more interests ${ }^{3-13}$. However, intense controversies on the causality issue of artificial active media still exist. Up to now, on the assertion whether there is a theoretical stable state for the simultaneous existence of negative refractive index (NRI) and net gain, physicists still have opposite opinions $^{14-19}$. In optics, a diverse family of 'gain media', primarily based on doped crystalline, semiconductors, dyes and gases, have been widely used in laser technologies ${ }^{20-24}$. Simulations have shown that by placing optical gain media (that is, optically pumped laser dyes) in fishnet metamaterial cells, the resulting optical 'gain metamaterial' is able to exhibit an NRI in a narrow bandwidth without violating causality ${ }^{4,7}$. The significance of this conception is that although this artificial gain medium still functions as a laser amplifier, thus compensating or even overcompensating the intrinsic loss of traditional metamateirals, it inherits the theoretical superiorities of metamaterials, being able to provide arbitrarily controlled constitutive parameters not found in nature ${ }^{5}$. Therefore, 'gain metamaterials' have the realistic potential in implementing metamaterial applications such as perfect lenses and invisibility cloaks, where intrinsic losses fundamentally plague their realizations. However, to our knowledge, although significant loss compensation for a fishnet metamaterial at optical frequency has been reported ${ }^{8}$, metamaterial with NRI and net gain has not been reported so far.

In this study, we experimentally demonstrate active metamaterial with simultaneous gain and negative index of refraction in microwave regime. We discuss the theory and implementation of an artificial microwave gain medium based on negative-resistance devices (that is, external energy pumping from direct current (DC) power supply). We derive the condition for achieving such a medium from the electromagnetic energy conservation in causal media. Equivalent circuit analysis, simulations and experimental measurements show that a fabricated sample consisting of traditional metamaterial building blocks with embedded microwave tunnel diodes (TDs) indeed exhibits a band-limited Lorentzian dispersion with overcompensation of loss and NRI. Our work provides experimental evidence to the discussion of the causality of negative-index gain medium, proposing a potential solution for the critical challenge current metamateiral technology faces in practical applications.

\section{Results}

Homogenization of effective gain medium. In Maxwell electromagnetic (EM) theory, the energy conservation of an EM wave propagating in a causal, naturally existing medium is described by Poynting's theorem ${ }^{25}$ :

$$
\nabla \cdot \mathbf{S}+i \omega \mathbf{E} \cdot(\varepsilon(\omega) \mathbf{E})^{*}-i \omega \mathbf{H}^{*} \cdot(\mu(\omega) \mathbf{H})=-\mathrm{J}^{*} \cdot \mathbf{E},
$$

where $\varepsilon(\omega)$ and $\mu(\omega)$ represent the causally dispersive permittivity and permeability, respectively, $\nabla \cdot \mathbf{S}$ is the divergence of complex Poynting vector, whose real part represents the total power flowing out an infinitesimally small closed volume, which is always negative for lossy passive media. The term $-\mathbf{J}^{*} \cdot \mathbf{E}$ in equation (1) may consist of a conductive current density $\mathbf{J}_{c}(\omega)=\sigma_{c}(\omega) \mathbf{E}(\omega)$, where $\sigma_{c}(\omega)$ is the causally dispersive conductivity, and a free current density $\mathbf{J}_{s}$. It should be noted that $\mathbf{J}_{s}=0$ in any passive medium. However, if $\mathbf{J}_{s}$ can be artificially provided homogeneously in the medium, and it can be controlled by the incident field, that is, $\mathbf{J}_{s}(\omega)=\sigma_{s}(\omega) \mathbf{E}(\omega)$, where $\sigma_{s}(\omega)$ is an effectively 'negative' conductance that is independent of $\mathbf{E}(\omega)$, equation (1) can be rewritten as

$$
\nabla \cdot \mathbf{S}+i \omega \mathbf{E} \cdot\left(\varepsilon_{e f f}(\omega) \mathbf{E}\right)^{*}-i \omega \mathbf{H}^{*} \cdot(\mu(\omega) \mathbf{H})=0
$$

where $\varepsilon_{\text {eff }}(\omega)=\varepsilon_{\text {eff }}^{\prime}(\omega)+i \varepsilon_{\text {eff }}^{\prime \prime}(\omega)=\varepsilon(\omega)+i\left[\sigma_{c}(\omega)+\sigma_{s}(\omega)\right] / \omega$ can be regarded as the effective permittivity of a new artificial medium. For a plane wave propagating in such an effective medium, assume $\mathbf{E}(\omega)=\hat{x} E_{0} e^{i k z}$ and $\mathbf{H}(\omega)=\hat{y} E_{0} e^{i k z} / \eta$, where $\quad k=k^{\prime}+i k=\omega \sqrt{\varepsilon_{e f f} \mu}$ and $\eta=\eta^{\prime}+i \eta=\sqrt{\mu / \varepsilon_{e f f}}$ are the complex wave number and wave impedance, respectively. Substitute $\mathbf{E}(\omega)$ and $\mathbf{H}(\omega)$ into equation (2), we obtain

$\operatorname{Re}[\nabla \cdot \mathbf{S}]=-\omega\left|\varepsilon_{e f f}(\omega)\right||\mathbf{E}|^{2}\left(\varepsilon_{e f f}^{\prime \prime}(\omega) /\left|\varepsilon_{e f f}(\omega)\right|+\mu^{\prime \prime}(\omega) /|\mu(\omega)|\right)$

For a sufficiently negative $\sigma_{s}(\omega), \quad \varepsilon_{e f f}^{\prime \prime}(\omega)=\left[\sigma_{c}(\omega)+\sigma_{s}(\omega)\right] / \omega$ can be negative, resulting in a positive $\operatorname{Re}[\nabla \cdot \mathbf{S}]$. In this case, the effective medium described by $\varepsilon_{\text {eff }}(\omega)$ can be macroscopically considered as a gain medium, providing a spatially uniform amplification to incident waves. It is worth noting that the dispersion of $\varepsilon_{\text {eff }}(\omega)$ is a linear superposition of the conventional complex permittivity $\varepsilon(\omega)+i \sigma_{c}(\omega) / \omega$ and $i \sigma_{s}(\omega) / \omega$, meaning that the causality of $\varepsilon_{\text {eff }}(\omega)$ fully depends on the dispersion of $\sigma_{s}(\omega)$. That said, from energy conservation (Poynting's theorem) point of view, if $\sigma_{s}(\omega)$ can be physically obtained, $\varepsilon_{e f f}(\omega)$ will consequently satisfy causality.

It is seen that the key to implement an artificial gain medium is to obtain a uniform spatial distribution of a negative conductance $\sigma_{s}(\omega)$ (or resistance). In microwave regime, this can be achieved by embedding negative-resistance devices, such as TDs, into conventional sub-wavelength metamaterial unit cells, as shown in Fig. 1. TDs are quantum tunnelling semiconductor devices capable of offering a negative differential resistance (NDR) at microwave frequencies ${ }^{6,26}$. This NDR can be equivalently considered as a negative resistance $-R_{\mathrm{d}}$, acting as a current source linearly controlled by an applied voltage. Similar NDR device was used to improve the quality factor of a split ring resonator (SRR) by partially compensating its loss ${ }^{11}$. In Fig. 1a, we show examples of embedded TD in sub-wavelength wire and SRR cells, which are the basic building blocks for constructing metamaterials.

For the wire array under a $z$-polarized plane wave incidence, the voltage across the sub-wavelength wires can be expressed as $U=E_{\mathrm{z}} d=R I+(-i \omega L) I+I /(-i \omega C)+U_{\mathrm{d}}$, where $I$ is the induced current, $R$ and $L$ are the total parasitic resistance and inductance, respectively, (including mutual inductance between adjacent wires) of the wire $\mathrm{r}^{27,28}, C$ is the total series capacitance caused by the open ends of the wire and the gap used to mount the TD and $U_{\mathrm{d}}=-I R_{\mathrm{d}}$ is the voltage across the TD. Then, the volume current density $J_{\mathrm{v}}$ in each cell can be homogenized as

$$
\begin{aligned}
J_{v} & =\frac{I}{d^{2}}=\frac{i \omega /(d L)}{\omega^{2}-1 /(L C)+i \omega\left(R-R_{d}\right) / L} E_{z}=J_{c}+J_{d} \\
& =\left(\sigma_{c}+\sigma_{d}\right) E_{z}
\end{aligned}
$$

where $d$ is the periodicity of the cell. According to equation (2), 
a

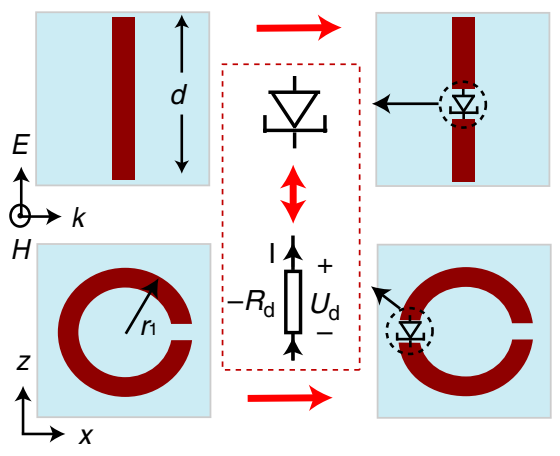

b

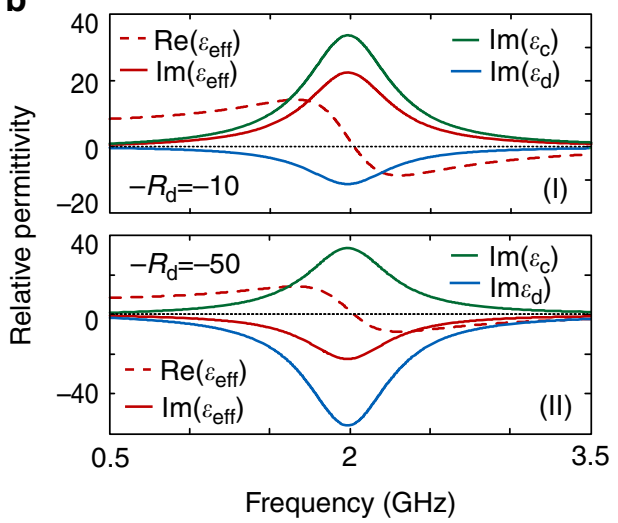

Figure 1 | Homogenization of microwave gain media. (a) Schematic diagrams of embedding TDs in sub-wavelength metallic wire and SRR, to achieve building blocks of gain metamaterials, where the TD is equivalent to a negative resistance $-R_{\mathrm{d}}$. (b) Effective permittivity of the metallic wire array calculated by equation (5). In the calculation, $d=0.02 \mathrm{~m}, R=30 \Omega, L=5 \mathrm{nH}, C=1.25 \mathrm{pF},-R_{\mathrm{d}}=-10 \Omega$ for I and $-R_{\mathrm{d}}=-50 \Omega$ for II. It is seen that when $-R_{\mathrm{d}}=-50 \Omega$, the effective medium is overcompensated and thus the calculated permittivity $\varepsilon_{\text {eff }}$ has a negative imaginary part (red solid line).

$\varepsilon_{\text {eff }}$ can be described by

$$
\begin{aligned}
\varepsilon_{e f f}= & 1+i \frac{\left[\sigma_{c}+\sigma_{d}\right]}{\omega \varepsilon_{0}}=1+\varepsilon_{c}+\varepsilon_{d} \\
\varepsilon_{c}= & \frac{-\left[\omega^{2}-1 /(L C)\right] /\left(d L \varepsilon_{0}\right)}{\left[\omega^{2}-1 /(L C)\right]^{2}+\left[\omega\left(R-R_{d}\right) / L\right]^{2}} \\
& +i \frac{\omega R /\left(d L^{2} \varepsilon_{0}\right)}{\left[\omega^{2}-1 /(L C)\right]^{2}+\left[\omega\left(R-R_{d}\right) / L\right]^{2}} \\
\varepsilon_{d}= & i \frac{-\omega R_{d} /\left(d L^{2} \varepsilon_{0}\right)}{\left[\omega^{2}-1 /(L C)\right]^{2}+\left[\omega\left(R-R_{d}\right) / L\right]^{2}}
\end{aligned}
$$

Note that the signs of the imaginary parts of $\varepsilon_{\mathrm{c}}$ and $\varepsilon_{\mathrm{d}}$ depend on the positive parasitic resistance $R$ and the negative resistance $-R_{\mathrm{d}}$ of the TD, respectively. In Fig. $1 \mathrm{~b}$, we plot the $\varepsilon_{\text {eff }}$ derived above. Relevant parameters are $d=0.02 \mathrm{~m}, R=30 \Omega, L=5 \mathrm{nH}$ and $C=1.25 \mathrm{pF}$, where $L$ and $C$ are determined based on refs 27,28 . It is seen that when $-R_{\mathrm{d}}$ is $-10 \Omega$, although the positive imaginary part of $\varepsilon_{\mathrm{c}}$ is compensated by $\varepsilon_{\mathrm{d}}$, the overall imaginary part of $\varepsilon_{e f f}$ remains positive so that the overall effective medium has a loss. However, when $-R_{\mathrm{d}}$ is $-50 \Omega$, overcompensation occurs and $\varepsilon_{\text {eff }}$ effectively has a negative imaginary part so that the overall effective medium has a gain. According to ref. 3, the real and imaginary parts of $\varepsilon_{\text {eff }}$ satisfy the Krammers-Kronig relations defined for active media, implying that this $\varepsilon_{\text {eff }}$ obeys causality.

In a similar way, we can also derive the effective permeability with a potentially negative imaginary part for the SRR array embedded with TDs as shown in Fig. 1a. Details about this derivation and the corresponding equation of $\mu_{\text {eff }}$ can be found in Methods section.

For a metamaterial comprising such wire and/or SRR cells, when $-R_{\mathrm{d}}$ is chosen appropriately so that the loss of the metamaterial can be overcompensated, an effective microwave gain medium with a unique frequency dispersion determined by the 'active' resonance of these sub-wavelength resonators, including a NRI, can be realized.

Full-wave simulation. For demonstration, we design a new single-layer cell that combines the wire and SRR resonators in the same plane as shown in Fig. 2a. Without losing any generality, such a composite wire-SRR cell only requires one active element for simplicity. The chosen TD is the General Electric's TD261, whose current-voltage characteristic and equivalent circuit is provided in Fig. 2b. In the NDR region, TD261 is effectively composed of a negative resistance $-R_{\mathrm{d}}$ and parasitic components $R_{\mathrm{p}}, L_{\mathrm{p}}$ and $C_{\mathrm{p}}$ due to the device package. Although the highfrequency limit of TD261 can be up to $20 \mathrm{GHz}$, to minimize the impact of the parasitic parameters, the resonance frequency of the unit cell is chosen to be around $2 \mathrm{GHz}$. In the design, the dimensions of the metallic pattern are optimized to align the electric and magnetic resonance regions, achieving double-negative bands of $\varepsilon_{e f f}$ and $\mu_{\text {eff }}$ within the same frequency range. Such metallic patterns are printed on a 1.27-mm-thick Rogers-6006 substrate with $\varepsilon_{\mathrm{r}}=6.5$ and the optimized dimensions are $a=27$ $\mathrm{mm}, b=54 \mathrm{~mm}, c=4 \mathrm{~mm}, d=5 \mathrm{~mm}, e=6 \mathrm{~mm}, f=0.8 \mathrm{~mm}$, $g=2.5 \mathrm{~mm}, r_{1}=9 \mathrm{~mm}$ and $r_{2}=12 \mathrm{~mm}$. At $2 \mathrm{GHz}$, the unit cell periodicity is $<\lambda / 5$. Note that such a periodicity can ensure a sufficiently homogeneous field distribution in the artificial medium, and therefore the obtained medium can be characterized by effective permittivity and permeability ${ }^{29}$. Then, the Simulation program with integrated circuit emphasis (SPICE) model and DC bias of the TD261 is introduced in the pattern, to simulate the compensation effect on the loss. According to the manufacturer's data, at a bias voltage of $0.2 \mathrm{~V}$, the TD261 parameters are: $R_{\mathrm{p}}=7 \Omega, L_{\mathrm{p}}=1.5 \mathrm{nH}$, $C_{\mathrm{p}}=0.65 \mathrm{pF}$ and $-R_{\mathrm{d}}=-250 \Omega$.

Figure 2c plots the simulated $S$ parameters of the metamaterial consists of such unit cells. A commercial finite-element Maxwell equation solver, high frequency structural simulator (HFSS), is used to perform the full-wave simulation for two cases, that is, with and without the TD. To implement a full-wave simulation for the metamaterial structure embedded with biased TD, the built-in field/circuit co-simulation of the solver is used. Using the homogenization approach proposed in refs 30,31, retrieved effective parameters are shown in Fig. 2d,e. From Fig. 2d, it can be seen that without the TD, the imaginary part of the retrieved refractive index is always positive. In the bandwidth of 1.97$2.1 \mathrm{GHz}$, the imaginary part is small and the corresponding real part is negative, exhibiting a similar dispersion of NRI as those reported in previous literature ${ }^{7}$. After including the TD and varying $-R_{\mathrm{d}}$ from -50 to $-250 \Omega$, it can be seen that in the upper inset of Fig. $2 \mathrm{~d}$ the imaginary part of the NRI becomes negative. The trend of these changes with the increased $-R_{\mathrm{d}}$ is consistent with the simulation results for optical gain medium reported in ref. 7 . In the lower inset of Fig. $2 \mathrm{~d}$, we also plot the dispersion of the real part of NRI for $-R_{\mathrm{d}}$ ranges from -50 to $-900 \Omega$. As indicated in equation (5), the value of $-R_{\mathrm{d}}$ has an impact on the real part of $\varepsilon_{\mathrm{c}}$, and thus $\varepsilon_{\text {eff. }}$. Therefore, when $-R_{\mathrm{d}}$ changes in a large range, the real part of NRI changes notably as well. 
a

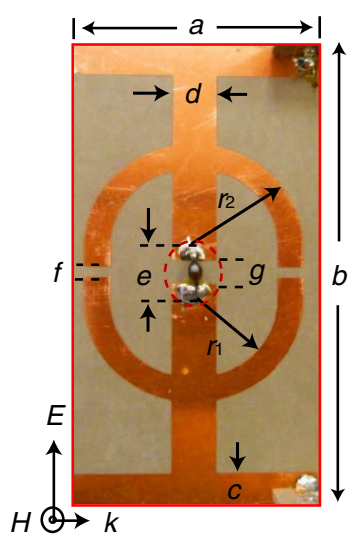

b

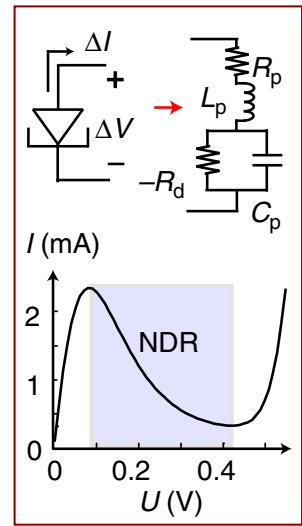

C

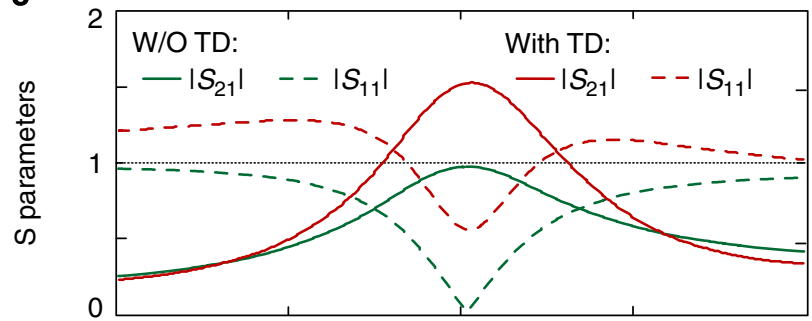

d
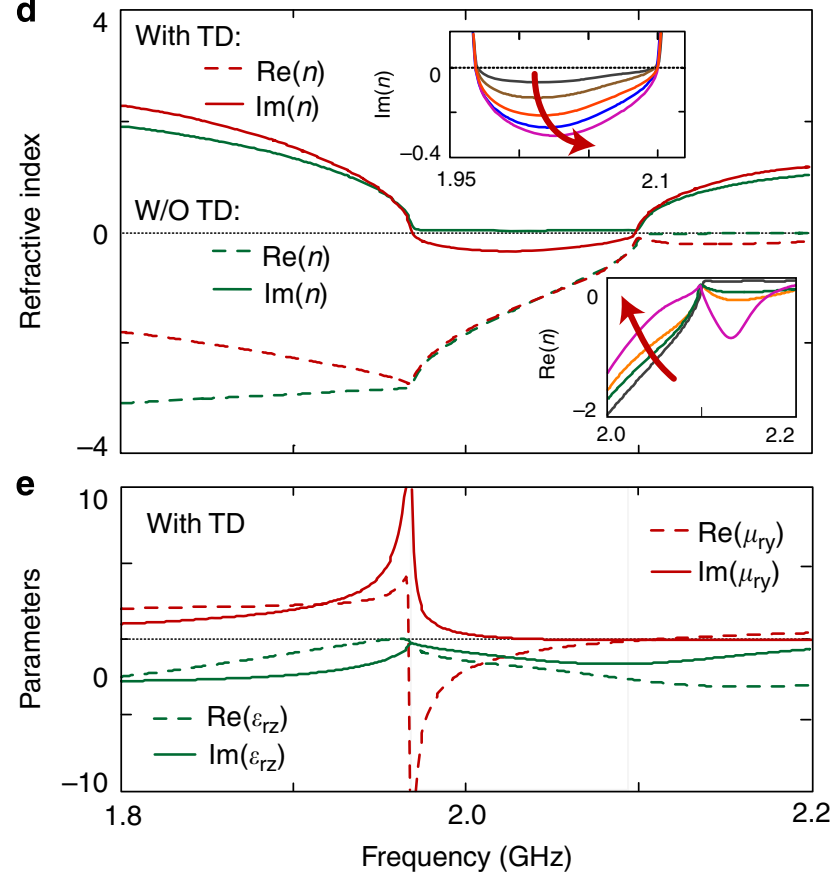

Figure 2 | Simulated results and retrieved parameters of the sub-wavelength wire and SRR-based artificial medium with and without embedded TDs. (a) Photograph of the fabricated unit cell with an embedded TD. Metallic patterns are printed on a 1.27-mm-thick Rogers-6006 substrate, whose dielectric constant is 6.5. Related dimensions are $a=27 \mathrm{~mm}, b=54 \mathrm{~mm}, c=4 \mathrm{~mm}, d=5 \mathrm{~mm}, e=6 \mathrm{~mm}, f=0.8 \mathrm{~mm}, g=2.5 \mathrm{~mm}, r_{1}=9 \mathrm{~mm}$ and $r_{2}=12 \mathrm{~mm}$. (b) Equivalent circuit model of the embedded TD (TD261 by General Electric) and its $I-V$ curve, where the grey area indicates the NDR region. $R_{p}, L_{p}$ and $C_{p}$ are the parasitic resistance, inductance and capacitance of the TD, respectively. (c) Simulated S parameters of the unit cell with and without the TD under a normal plane wave incidence. The polarization of the incidence is shown at the bottom left corner of $\mathbf{a}$. (d) Retrieved effective refractive index of the effective medium with and without TD. The upper and lower insets show the dependency of the real and imaginary parts of retrieved refractive index on $-R_{\mathrm{d}}$. As indicated by the red arrows, with an increased $R_{\mathrm{d}}$, while the imaginary part of the refractive index becomes more negative, the real part becomes less negative. (e) Retrieved constitutive parameters of the effective medium with embedded TD.

For further analysis, we show the retrieved constitutive parameters in Fig. 2e. It is observed that after introducing the TD, the real parts of $\varepsilon_{r z}$ and $\mu_{r y}$ are both negative within an overlapped bandwidth from 1.97 to $2.1 \mathrm{GHz}$. It is worth noting that although the imaginary part of permittivity is always negative, the imaginary part of permeability is positive. As the TD is incorporated on the wire resonator, it mainly provides a current amplification to the electric resonance, rather than the magnetic resonance of the SRR. Nevertheless, one can imagine flexible designs tailoring the electric and the magnetic responses independently by judiciously placing TDs.

To further confirm that the demonstrated metamaterial is effectively a negative indexed gain medium, full-wave simulations of a prism-like sample made of such a metamaterial and the corresponding air prism reference are performed. As shown in Fig. 3a, the prism consists of 10 unit cells $(270 \mathrm{~mm})$ along the $y$ direction, and 5 unit cells $(135 \mathrm{~mm})$ and 2 unit cells $(54 \mathrm{~mm})$ along the $x$ direction on the two sides of the prism, respectively. The angle between the $x$ direction and the normal of the refraction interface is $26.6^{\circ}$ as shown in Fig. 3b. In the simulation setup, the prism is placed between two parallel perfect conducting plates separated by $54 \mathrm{~mm}$, which equals the thickness of the metamaterial. A $z$-polarized plane wave is generated to transmit along a parallel-plate waveguide and is normally incident on the prism. A semicircular free space chamber with a radius of $270 \mathrm{~mm}$ is used to observe the refracted wave.

Figure $3 \mathrm{a}$ shows the simulation result when the incident frequency is $1.948 \mathrm{GHz}$ and $-R_{\mathrm{d}}$ is $-260 \Omega$. It can be clearly observed that the refracted wave is on the same side of the normal as the incident wave. With a refractive angle of $-17.4^{\circ}$, the corresponding NRI of the prism is about -0.67 . For comparison, Fig. $3 \mathrm{~b}$ shows the result in the absence of the prism, or equivalent to an 'air prism'. It is observed that although the refractive angle is positive at $26.6^{\circ}$, the transmission power is lower than that shown in Fig. 3a. To observe the gain of the metamaterial prism more quantitatively, we also plot the far-field radiation patterns of these two cases in Fig. 3c. Compared with the 'air prism', not only the radiation pattern of the metamaterial prism points to the direction of $44^{\circ}$ (corresponding to a refractive angle of $-17.4^{\circ}$ ) but also the gain of the metamaterial prism is $2.37 \mathrm{~dB}$ higher, even though it has a worse impedance matching at the incident interface between the parallel-plate waveguide and the prism. 

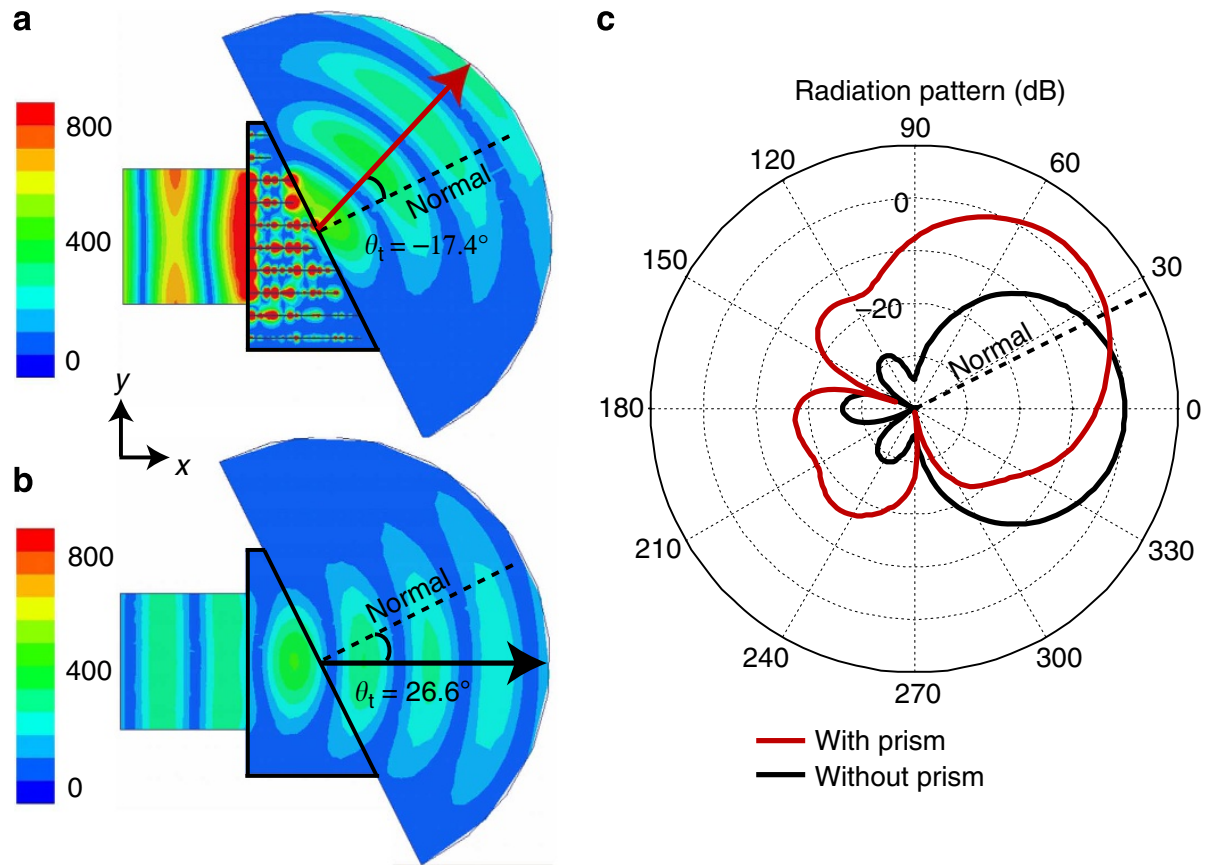

Figure 3 | Full-wave simulations. (a) Simulated electric field distribution under a normal incidence to a designed prism-like sample. The incident frequency is $1.948 \mathrm{GHz}$ and the electric field is polarized along the $z$ axis. In the simulation, the prism comprises 10 unit cells $(270 \mathrm{~mm})$ along the $y$ direction, and 5 unit cells $(135 \mathrm{~mm})$ and 2 unit cells $(54 \mathrm{~mm})$ along the $x$ direction on the two sides of the prism, respectively. The incident angle on the slope side is $26.6^{\circ}$ and the simulated refractive angle is $-17.4^{\circ}$. (b) An air prism with identical size as a reference. It is seen that the transmitted wave keeps its direction unchanged as the incidence. (c) Simulated far-field radiation patterns for cases $\mathbf{a}$ (red line) and $\mathbf{b}$ (black line). The radiation patterns point to the same directions as those in $\mathbf{a}$ and $\mathbf{b}$, respectively. Compared with the air prism, a relative gain of $2.37 \mathrm{~dB}$ is observed for the gain metamaterial prism.

Note that owing to the presence of higher-order Bloch modes in multilayer metamaterials ${ }^{32}$, the frequency $1.948 \mathrm{GHz}$ is slightly lower than the NRI bandwidth shown in Fig. 2.

Experimental measurement. Finally, we use the standard rectangular waveguide method to experimentally characterize the effective constitutive parameters of the fabricated sample of the designed metamaterial. The measurement setup is shown in Fig. $4 \mathrm{a}$, where a standard WR-430 waveguide with a cross-section of $109.22 \mathrm{~mm} \times 54.61 \mathrm{~mm}$ is used to hold the fabricated sample. According to ref. 33, a single-layer sample consisting of 4 unit cells is used in the parameter retrieval to achieve a higher accuracy. Thin enameled copper wires are used to apply the bias voltage to the TDs. A pre-test has been performed to verify that such a biasing method does not influence the microwave propagation in the waveguide. Three sets of measurement are performed, that is, the sample without the TDs soldered on, the sample with unbiased TDs and the sample with TDs biased at a DC voltage of $0.38 \mathrm{~V}$. For each configuration, $S$ parameters are measured using an Agilent's E8361A vector network analyser in the frequency band of $1.8-2.2 \mathrm{GHz}$. Note that in this band, only the $\mathrm{TE}_{10}$ mode can exist in the WR-430 waveguide.

Figure $4 \mathrm{~b}$ plots the measured $S$ parameters for the first and third configurations. It is seen that for the no TDs case, the measured $S$ parameters are consistent with the simulated results shown in Fig. 2c, except a slightly shifted frequency $(1.93 \mathrm{GHz})$ of the dip of $S_{11}$, which indicates impedance matching. For the biased TDs case, although the dip of $S_{11}$ can still be observed at an adjacent frequency $(1.96 \mathrm{GHz})$, the amplitude of $S_{21}$ is higher than unity in the vicinity, showing a nearly $3.5 \mathrm{~dB}$ gain at $1.98 \mathrm{GHz}$. It is worth noting that in the bandwidth with net gain, the measured $S_{21}$ curve has observable noises that are larger than the case without TDs. According to ref. 34, these noises are possibly induced by the introduction of the net gain through the fluctuation-dissipation theorem.

Figure $4 \mathrm{c}$ plots the retrieved refractive indices for all three configurations. In the frequency band of $1.92-2 \mathrm{GHz}$, it can be seen that for both the no TDs and the unbiased TDs cases, the sample exhibits an NRI with a relatively small positive imaginary part. However, for the biased TDs case, although the sample exhibits a NRI, it also has a negative imaginary part. It is also worth noting that the measured real part of the refractive index looks similar to the curve in the lower inset of Fig. $2 \mathrm{~d}$ when $-R_{\mathrm{d}}=-900 \Omega$. As the net gain and the NRI band are observed when the DC bias is $0.38 \mathrm{~V}$, according to the $I-V$ curve of the TD in Fig. 2b, such a DC bias is close to the upper boundary of the NDR region. In this case, the corresponding $-R_{\mathrm{d}}$ does have a large negative value, which is consistent to the simulation. Compared with the measured result without TDs, a frequency shift of the negative band can be observed for the curves with TDs, which is obviously due to the parasitic reactance of the TDs. These experimental results agree well with the simulation results.

To make sure there is no self-resonance in the $S$ parameter measurements for the biased TDs case, the input power is swept. It is observed that when the incident power is swept from -60 to $-45 \mathrm{dBm}$, the measured results keep the same, exhibiting a linear amplification around $+3.5 \mathrm{~dB}$ in the entire power range. However, when the incident power becomes higher than $-45 \mathrm{dBm}$, the gain starts to decrease, implying nonlinearity begins to set in and dominate, as shown in the inset of Fig. 4c. Therefore, the power dependence results confirm that when the input power is less than $-45 \mathrm{dBm}$, this active microwave metamaterial is in the linear range.

\section{Discussion}

In conclusion, we discuss the theory and implementation of an artificial microwave gain medium incorporating negative-resistance 
a

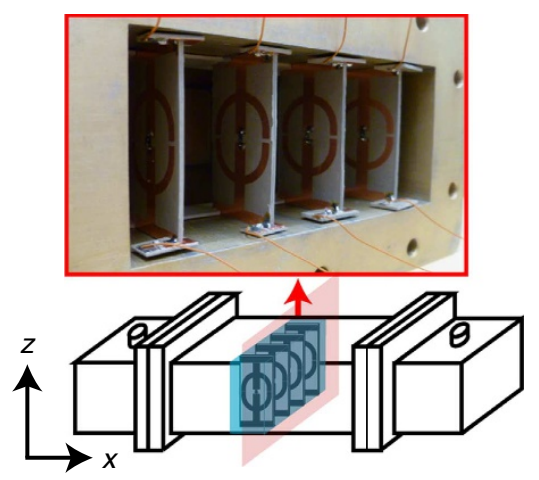

b

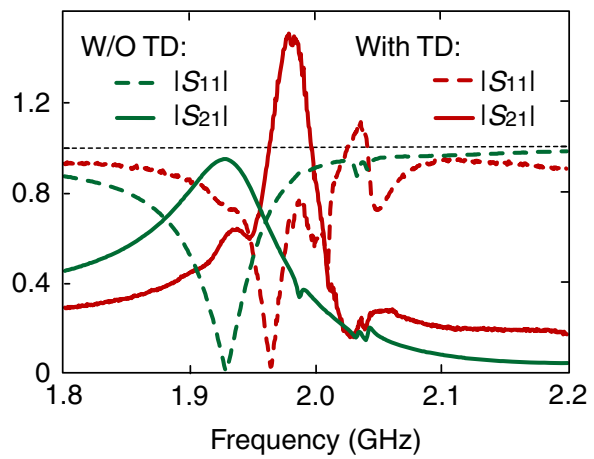

c

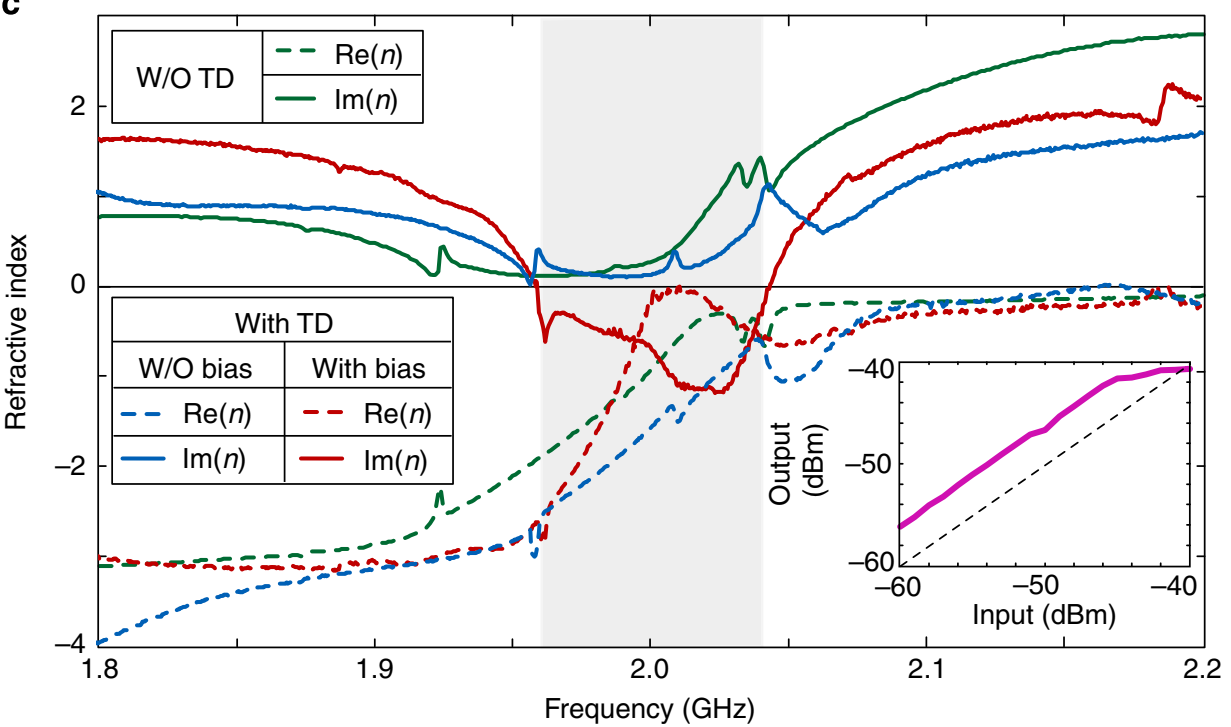

Figure 4 | Experimental results and retrieved parameters of the fabricated metamaterial sample. (a) Photograph (top) and schematic drawing (bottom) of the fabricated single-layer sample placed in a standard WR-430 rectangular waveguide. (b) Measured S parameters for the samples without TDs (green lines) and with TDs biased at a DC voltage of $0.38 \mathrm{~V}$ (red lines). The $S$ parameters were measured using a vector network analyser, that is, Agilent E8361a. (c) Retrieved refractive indices from the experimental S parameters for three different configurations, that is, the sample without TDs (green lines), the sample with unbiased TDs (blue lines) and the sample with biased TDs (red lines). The inset shows the dependency of the output power on the input power. It is seen that in a wide range of incident power, the gain remains stable.

devices. Based on the causality inherently described by the EM energy conservation, we derive the condition for achieving such a medium from Poynting's theorem. Equivalent circuit analysis, simulations and experimental measurements show that a fabricated sample consisting of traditional metamaterial building blocks with microwave TDs embedded in the unit cells exhibits a band-limited Lorentzian dispersion with an overcompensated loss (gain) and an NRI. This result provides an experimental evidence that supports refs 4,17,19. Meanwhile, considering that the inevitable loss due to the strongly dispersive nature of artificial metamaterials is one of the fundamental challenges preventing many interesting applications from becoming a reality, the proposed microwave gain medium can be effectively used to solve this problem. By incorporating state-of-the-art semiconductor and micro-nanofabrication technologies, solid-state gain medium with NRI can be obtained. Similar to the optical laser gain media, the implementation of effective gain media in microwave domain would have significant potentials in microwave engineering. This kind of active metamaterials may be useful not only in designing and achieving new and high-performance microwave circuits and antennas, but also as a simple model system to study fundamental physics of active metamaterials in general and shed light on metamaterial applications at other spectra, including optical frequencies. In fact, terahertz NDR diodes and short optical pulse-pumped organic dye molecules and quantum dots that exhibit effective NDR properties have been reported ${ }^{7,35,36}$, implying the possibility to implement such artificial gain media at terahertz and optical frequencies.

\section{Methods}

Homogenization of an effective gain medium composed of SRRs. In this section, we demonstrate the equivalent circuit analysis based homogenization of an artificial gain medium with negative permeability, which comprises traditional SRRs with microwave TDs embedded. For convenience, we also use an effective negative resistance $-R_{\mathrm{d}}$ to represent the TD, as shown in the bottom panel of Fig. 1a.

We obtain the effective permeability of this metamaterial based on the same equivalent circuit analysis proposed in ref. 37. Considering an infinite array of such SRRs arranged in three orthogonal directions with a spatial period of $d$, and an incident magnetic field polarized along the $y$ direction, that is, perpendicular to the SRRs, there would be electromotive force (EMF) and induced current $I$ along the rings, satisfying EMF $=i \omega \pi r^{2} \mu_{0} H_{0}=R I+I /(-i \omega C)+$ $(-i \omega L) I+(-i \omega F L) I+U_{\mathrm{d}}$, where $R$ and $L$ are the parasitic resistance and the geometrical inductance of each ring, respectively, $F L$ is the mutual inductance 
between different rings. With $L=\pi r^{2} \mu_{0} / d, F=\pi r^{2} / d^{2}$ (ref. 37), the current $I$ is

$$
I=\frac{-i \omega \pi r^{2} \mu_{0} H_{0}}{i \omega \pi r^{2} \mu_{0}\left[1-\frac{\pi r^{2}}{d^{2}}\right]-R+R_{d}+\frac{1}{i \omega C}}=\frac{-d H_{0}}{\left(1-F-\frac{1}{\omega^{2} L C}\right)+\frac{R i}{\omega L}+\frac{-R_{d} i}{\omega L}}
$$

Based on the magnetic moment per unit volume $M=\pi r^{2} I / d^{3}$ and $\mu_{\text {eff }}=\left(B / \mu_{0}\right) /(B /$ $\left.\mu_{0}-M\right)$, we obtain the final effective permeability as follows:

$$
\begin{aligned}
& \mu_{e f f}=1-\frac{F}{\left(1-\frac{1}{\omega^{2} L C}\right)+\frac{R i}{\omega L}+\frac{-R_{d} i}{\omega L}}=1+\mu_{c}+\mu_{d} \\
& \mu_{c}=\frac{-F\left(1-\frac{1}{\omega^{2} L C}\right)+F R i / \omega L}{\left[\left(1-\frac{1}{\omega^{2} L C}\right)^{2}+\left(\frac{R-R_{d}}{\omega L}\right)^{2}\right]} \\
& \mu_{d}=\frac{-F R_{d} i / \omega L}{\left[\left(1-\frac{1}{\omega^{2} L C}\right)^{2}+\left(\frac{R-R_{d}}{\omega L}\right)^{2}\right]}
\end{aligned}
$$

Similar to the permittivity case, we find that the sign of the imaginary parts of $\mu_{\mathrm{c}}$ and $\mu_{\mathrm{d}}$ depends on the positive parasitic resistance $R$ and the negative resistance $R_{\mathrm{d}}$ of the TD. A negative imaginary part of $\mu_{\text {eff }}$ would appear when $R_{\mathrm{d}}>R$, meanwhile the real part of $\mu_{\text {eff }}$ still exhibits a traditional Lorentzian dispersion.

\section{References}

1. Pendry, J. B. Negative refraction makes a perfect lens. Phys. Rev. Lett. 85, 3966 (2000).

2. Schurig, D. et al. Metamaterial electromagnetic cloak at microwave frequencies. Science 314, 977-980 (2006).

3. Fang, A., Koschny, T. \& Soukoulis, C. M. Self-consistent calculations of losscompensated fishnet metamaterials. Phys. Rev. B 82, 121102 (2010).

4. Hamm, J. M., Wuestner, S., Tsakmakidis, K. L. \& Hess, O. Theory of light amplification in active fishnet metamaterials. Phys. Rev. Lett. 107, 167405 (2011).

5. Hess, O. et al. Active nanoplasmonic metamaterials. Nat. Mater. 11, 573-584 (2012).

6. Jiang, T., Chang, K. H., Si, L., Ran, L. \& Xin, H. Active microwave negativeindex metamaterial transmission line with gain. Phys. Rev. Lett. 107, 205503 (2011).

7. Wuestner, S., Pusch, A., Tsakmakidis, K. L., Hamm, J. M. \& Hess, O. Overcoming losses with gain in a negative refractive index metamaterial. Phys. Rev. Lett. 105, 127401 (2010).

8. Xiao, S. et al. Loss-free and active optical negative-index metamaterials. Nature 466, 735-736 (2010).

9. Hess, O. \& Tsakmakidis, K. L. Metamaterials with quantum gain. Science 339, 654-655 (2013).

10. Chen, P. Y., Argyropoulos, C. \& Alu, A. Broadening the cloaking bandwidth with non- foster metasurfaces. Phys. Rev. Lett. 111, 233001 (2013).

11. Xu, W., Padilla, W. J. \& Sonkusale, S. Loss compensation in metamaterials through embedding of active transistor based negative differential resistance circuits. Opt. Express 20, 22406-22411 (2012).

12. Boardman, A. D., Rapoport, Y. G., King, N. \& Malnev, V. N. Creating stable gain in active metamaterials. J. Opt. Soc. Am. B 24, A53-A61 (2007).

13. Yuan, Y., Popa, B. I. \& Cummer, S. A. Zero loss magnetic metamaterials using powered active unit cells. Opt. Express 17, 16135-16143 (2009).

14. Stockman, M. I. Criterion for negative refraction with low optical losses from a fundamental principle of causality. Phys. Rev. Lett. 98, 177404 (2007).

15. Kinsler, P. \& McCall, M. W. Causality-based criteria for a negative refractive index must be used with care. Phys. Rev. Lett. 101, 167401 (2008).

16. Stockman, M. I. Stockman replies. Phys. Rev. Lett. 107, 259702 (2011).

17. Pendry, J. B. \& Maier, S. A. Comment on "spaser action, loss compensation, and stability in plasmonic systems with gain". Phys. Rev. Lett. 107, 259703 (2011).

18. Stockman, M. I. Spaser action, loss compensation, and stability in plasmonic systems with gain. Phys. Rev. Lett. 106, 156802 (2011).

19. Wuestner, S., Pusch, A., Tsakmakidis, K. L., Hamm, J. M. \& Hess, O. Comment on "spaser action, loss compensation, and stability in plasmonic systems with gain”. Phys. Rev. Lett. 107, 259701 (2011).
20. Capua, A., Karni, O., Eisenstein, G., Reithmaier, J. P. \& Yvind, K. Extreme nonlinearities in InAs/InP nanowire gain media: the two-photon induced laser. Opt. Express 20, 5987-5992 (2012).

21. Kemp, A. J. et al. Thermal management in disc lasers: doped-dielectric and semiconductor laser gain media in thin-disc and microchip formats. J. Mod. Optics 54, 1669-1676 (2007).

22. Paschotta, R. Laser gain media: a diverse family of materials. Photon. Spectra 47, 57-60 (2013).

23. Pirri, A., Toci, G. \& Vannini, M. Characterization and comparison of $1 \%$ at $\mathrm{Yb}$-doped $\mathrm{Lu} 2 \mathrm{O} 3$ and $\mathrm{Sc} 2 \mathrm{O} 3$ ceramics as laser gain media. Laser Phys. 22, 1851-1855 (2012).

24. Tanaka, M. et al. X-ray laser beam with diffraction-limited divergence generated with two gain media. Opt. Lett. 28, 1680-1682 (2003).

25. Kong, J. A. Electromagnetic Wave Theory (EMW Pub, 2000).

26. Todd, C. D. Measurement of tunnel diode negative resistance. Rev. Sci. Instrum 32, 338-342 (1961).

27. Maslovski, S. I., Tretyakov, S. A. \& Belov, P. A. Wire media with negative effective permittivity: a quasi-static model. Microw. Opt. Techn. Lett. 35, 47-51 (2002).

28. Pendry, J. B., Holden, A. J., Stewart, W. J. \& Youngs, I. Extremely low frequency plasmons in metallic mesostructures. Phys. Rev. Lett. 76, 4773 (1996).

29. Shelby, R. A., Smith, D. R. \& Schultz, S. Experimental verification of a negative index of refraction. Science 292, 77-79 (2001).

30. Chen, X., Grzegorczyk, T. M., Wu, B. I., Pacheco, J. \& Kong, J. A. Robust method to retrieve the constitutive effective parameters of metamaterials. Phys. Rev. E 70, 016608 (2004).

31. Smith, D.R., Schultz, S., Markos, P. \& Soukoulis, C.M. Determination of effective permittivity and permeability of metamaterials from reflection and transmission coefficients. Phys. Rev. B 65, 195104 (2002).

32. Rockstuhl, C. et al. Transition from thin-film to bulk properties of metamaterials. Phys. Rev. B 77, 035126 (2008).

33. Chen, H. et al. Experimental retrieval of the effective parameters of metamaterials based on a waveguide method. Opt. Express 14, 12944-12949 (2006).

34. Pusch, A., Wuestner, S., Hamm, J. M., Tsakmakidis, K. L. \& Hess, O. Coherent amplification and noise in gain-enhanced nanoplasmonic metamaterials: a maxwell-bloch langevin approach. ACS Nano 6, 2420-2431 (2012).

35. Hess, O. \& Gehrig, E. Photonics of Quantum-Dot Nanomaterials and Devices: Theory and Modelling (Imperial College Press, 2011).

36. Lu, J. T. \& Cao, J. C. Terahertz generation and chaotic dynamics in GaN NDR diode. Semicond. Sci. Technol. 19, 451-456 (2004).

37. Pendry, J. B., Holden, A. J., Robbins, D. J. \& Stewart, W. J. Magnetism from conductors and enhanced nonlinear phenomena. IEEE Trans. Microw. Theory Techn. 47, 2075-2084 (1999).

\section{Acknowledgements}

This work is in part supported by the AFOSR under grants FA-9550-09-1-0567 and FA9550-13-1-0209, the China Postdoctoral Science Foundation under grant 2014M550325 and the NSFC under grants 61131002 and 61401393.

\section{Author contributions}

D.Y., L.R. and H.X. designed the research; D.Y. and K.C. performed the research. All authors contributed to data interpretation and the composition of the manuscript.

\section{Additional information}

Competing financial interests: The authors declare no competing financial interests.

Reprints and permission information is available online at http://npg.nature.com/ reprintsandpermissions/

How to cite this article: Ye, D. et al. Microwave gain medium with negative refractive index. Nat. Commun. 5:5841 doi: 10.1038/ncomms6841 (2014). 